\title{
Imaging limbal and scleral vasculature using Swept Source Optical Coherence Tomography
}

\author{
Ireneusz Grulkowski, ${ }^{1}$ Jonathan J. Liu, ${ }^{1}$ Bernhard Baumann, ${ }^{1}$ Ben Potsaid,,${ }^{1,2}$ Chen Lu, ${ }^{1}$ and James G. Fujimoto ${ }^{* 1}$ \\ ${ }^{I}$ Massachusetts Institute of Technology, Research Laboratory of Electronics, Dept. of Electrical Engineering and \\ Computer Science, 77 Massachusetts Ave, Cambridge MA 02139, USA \\ ${ }^{2}$ Advanced Imaging Group, Thorlabs Inc., 435 Route 206 North, Newton NJ 07860, USA
}

Received December 19, 2011; accepted December 20, 2011; published December 31, 2012

\begin{abstract}
We demonstrate an application of high-speed swept source optical coherence tomography for vessel visualization in the anterior segment of the human eye. The human corneo-scleral junction and sclera was imaged in vivo. Imaging was performed using a swept source OCT system operating at the $1050 \mathrm{~nm}$ wavelength range and $100 \mathrm{kHz}$ A-scan rate. High imaging speed enables the generation of 3D depth-resolved vasculature maps. The vessel visualization method revealed a rich vascular system in the conjunctiva and episclera.
\end{abstract}

Optical coherence tomography (OCT) is a low coherence interferometric imaging modality that can perform highspeed and high-resolution cross-sectional and volumetric imaging [1, 2]. Because it is non-invasive, high speed and high resolution, OCT is widely used as a standard procedure in clinical ophthalmology for retinal as well as anterior segment disease diagnosis [3-5].

Since the introduction of OCT, there has been tremendous progress in the performance of OCT instruments, regarding their speed and resolution, which has also been supported by the development of new processing methods enabling better visualization of microstructures [6]. Conventional Time-domain OCT systems use an interferometer with a mechanically scanned optical reference path. In Fourier domain OCT, interferograms (spectral fringes) are recorded either by a spectrometer with a line-scan camera (Spectral OCT) or by a single detector with a swept laser source (Swept Source OCT).

While anterior segment imaging was originally demonstrated using Time-domain OCT [7], a wide range of Fourier-domain OCT systems operating at different wavelengths for anterior segment imaging have since been reported [8-10]. Studies of the anterior segment using high-resolution OCT have demonstrated the clinical utility of OCT for diagnosis of corneal diseases as well as monitoring surgical outcome.

Generally, most anterior segment structures of the eye are considered avascular. The exception is the transition region between cornea and sclera called the limbus. This region contains vessels that are associated with the circulatory system, lymphatic system and aqueous

*E-mail: jgfuji@mit.edu, igrulkowski@fizyka.umk.pl outflow system. Their role includes cell nutrition, cell waste removal as well as maintaining the balance between production and drainage of aqueous humor. The disruption of aqueous flow dynamics is often regarded as one of the factors contributing to glaucoma development [11]. Clinical angiographic techniques require injecting fluorescent dye (indocyanine green or fluorescein) and photographing dye passing through the vessels. Visualization of lymph vessels is also very invasive procedure and is based on injecting fluorescent or radioactive compound.

The development of OCT processing methods which are sensitive to slow flow velocities combined with hardware modifications allows visualization of the retinal capillary network [12]. Small animal studies have shown that advanced processing techniques enable quantitative characterization of different vascular networks, including contrast-free visualization of lymphatic system [13]. Additionally, recent reports have shown the potential use of these techniques in anterior segment studies [14]. Kagemann et al. presented velocity measurements in the limbal regions [15]. Follow on studies showed 3-D outflow structures in human cadaver eyes during perfusion [16]. Recently, a technique known as ultrahigh sensitive optical microangiography (OMAG) has been used to perform microvascular imaging of limbal vessels [17]. However, most of these studies have been performed using Spectral OCT systems. Swept source OCT at $1050 \mathrm{~nm}$ has better performance compared with Spectral OCT in terms of deeper tissue penetration, longer imaging range and higher imaging speeds $[9,18]$.

In this letter, we demonstrate visualization of limbal and scleral vascular systems using a swept source OCT system operating at the $1050 \mathrm{~nm}$ wavelength range. In vivo imaging was performed. An intensity based visualization technique was utilized to distinguish vascular structures.

The scheme of a prototype fiber-based OCT system used in this study is shown in Fig. 1. The light source was a commercially available 1050nm swept-source laser (Axsun) operating with a sweep repetition rate of $100 \mathrm{kHz}$. The light was coupled into a sample arm and single-pass 
reference arm. The detection of interferometric fringes was performed by a high-speed $200 \mathrm{MHz}$ dual-balanced detector (Thorlabs), and the signal acquisition was performed with an optically clocked data acquisition card (Innovative Integration, X5-400M). The system provided axial and transverse resolutions of $7 \mu \mathrm{m}$ and $18 \mu \mathrm{m}$, respectively. The depth imaging range determined by detection hardware bandwidths and was $3.82 \mathrm{~mm}$ in the air. Sensitivity roll-off over the entire axial measurement range was $4 \mathrm{~dB}$. The incident power on the cornea was $1.85 \mathrm{~mW}$, which was within the safe exposure limit as determined by the American National Standards Institute (ANSI). The system sensitivity was measured to be $97 \mathrm{~dB}$.

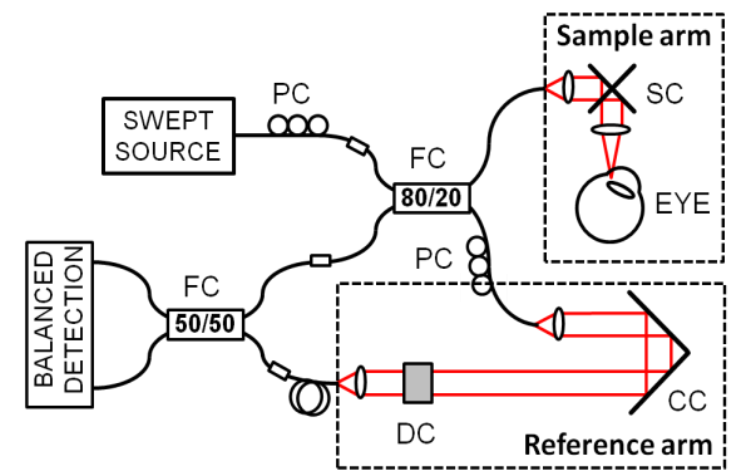

Fig. 1. Swept source OCT system. PC - polarization controller, FC fiber coupler, $\mathrm{SC}$ - galvanometric scanners, $\mathrm{CC}-$ corner cube, DC dispersion compensation.

Several scanning protocols were used as summarized in Table 1.

Table 1. Scanning protocols used in the experiments

\begin{tabular}{|c|c|c|c|c|}
\hline $\begin{array}{c}\text { Proto- } \\
\text { col }\end{array}$ & $\begin{array}{c}\text { Orien- } \\
\text { tation }\end{array}$ & $\begin{array}{c}\text { A-scans / } \\
\text { B-scans }\end{array}$ & $\begin{array}{c}\text { Scanning area } \\
{[\mathrm{mm} \times \mathrm{mm}]}\end{array}$ & $\begin{array}{c}\text { Total } \\
\text { measurement } \\
\text { time }[\mathrm{s}]\end{array}$ \\
\hline $\mathrm{A}$ & X-fast & $5000 / 5$ & $11.1 \times 0$ & 0.25 \\
\hline $\mathrm{B}$ & X-fast & $5000 / 5$ & $3.3 \times 0$ & 0.25 \\
\hline $\mathrm{C}$ & Y-fast & $300 / 300$ & $3.3 \times 2.2$ & 0.9 \\
\hline $\mathrm{D}$ & X-fast & $500 / 500$ & $5.3 \times 5.3$ & 2.5 \\
\hline
\end{tabular}

We applied an intensity-based angiographic method for visualization of vasculature in sclera images. The algorithm enhanced the differences in intensity images due to light attenuation in blood vessels (vessel shadowing effect). Before applying the algorithm, segmentation was performed to flatten the dataset relative to the scleral interface. Flattened images served as input for further algorithm steps that included thresholding and colorscale inversion. Finally, en face projections were generated.

Figure 2(a) presents cross-sectional images of the limbal and scleral region of the eye. The scanning protocols with repeated high definition B-scans were used (A and B from Table 1) and frame averaging was applied to generate the image in order to reduce speckle noise. The corneo-scleral

junction can be identified since scleral tissue is more scattering than corneal tissue. Vertical stripes across the image come from the vascular system present in the sclera (blood vessel shadowing effect). Enhanced penetration of light at the $1050 \mathrm{~nm}$ wavelength range enables visualization of deeper tissue structures such as the ciliary body. The general shape of the ciliary body can be easily seen. In addition, the pigmented posterior layer of the iris is highly scattering and can be easily distinguished. This layer is continuous with the retinal pigment epithelium in the posterior part of the eye.
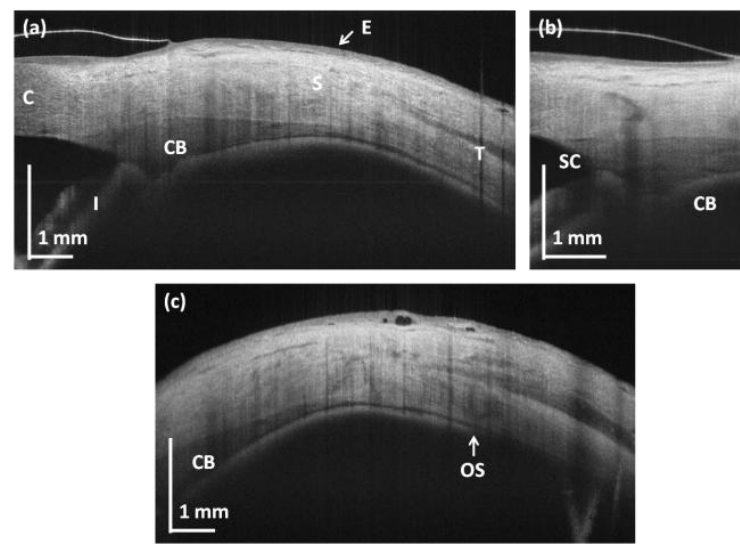

Fig. 2. OCT cross-sections of the human corneo-scleral region. (a) Structural high-definition scan (protocol A); (b) limbus (protocol B); (c) peripheral sclera (protocol A). C - cornea, S - sclera, E- epithelium, I - iris, SC - Schlemm's canal, OS - ora serrata.

We also performed 3-D imaging of limbal structures. The rendering and example cross-sections in all three dimensions are shown in Fig. 3. High-resolution enables visualization of Schlemm's canal [Fig. 3(c)]. Since the aqueous is a clear and non-scattering fluid, it appears as a dark band in the projection image [Fig. 3(b)].
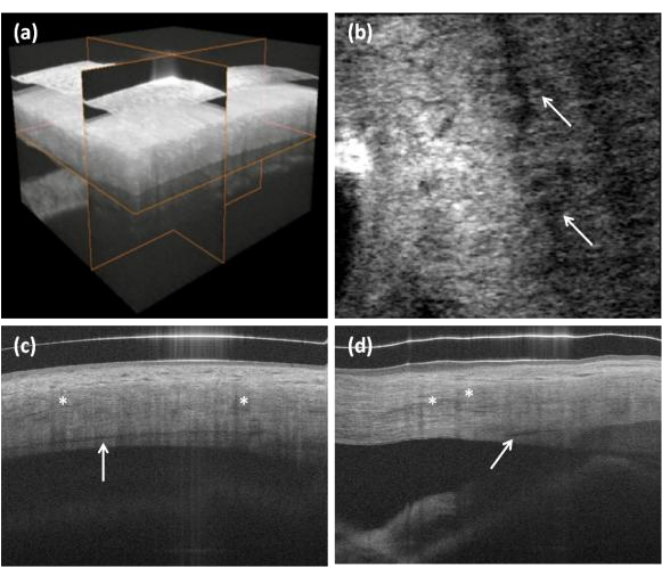

Fig. 3. In vivo 3-D imaging of human limbus. (a) rendering and 3-D reconstruction of data set. (b) $\mathrm{C}$-scan (x-y cross section). Arrows show Schlemm's canal. (c) B-scan in fast scanning axis ( $y-z$ cross-section). (d) $\mathrm{B}$-scan in slow scanning axis ( $\mathrm{x}-\mathrm{z}$ cross section). Protocol $\mathrm{C}$ from Table 1 was used for scanning the eye. Asterisks show blood vessel shadows. 
Blood vessels in OCT structural images can be recognized as areas with reduced speckle contrast. Since swept source OCT does not suffer from fringe wash-out as spectral OCT does, there is only light attenuation due to strong absorption of the light that contributes to characteristics of vessels in OCT images. As a consequence, structures beneath blood vessels are shadowed. There is therefore high certainty that empty spaces with no signal come from a non-scattering medium such as lymph or aqueous. These vessels do not produce any shadows since no absorption occurs [compare Schlemm's canal in Figs. 3(c)-(d)].

Following the algorithm described above we also generated projection images of vasculature (Fig. 4). The peripheral part of the sclera was imaged and the interface of tissue was segmented. After that, the entire data set was flattened to perform depth sectioning [Fig. 4(a)]. Projection images from data at different depths are shown in Fig. 4. Different layers contain different vasculature networks. The superficial vascular plexus of the conjunctiva is shown in Fig. 4(a). The large empty spaces are cysts which may result from contact lenses wearing. Cystic structures may be also related to the natural aqueous regulatory system. The deeper layers show different vasculature patterns.
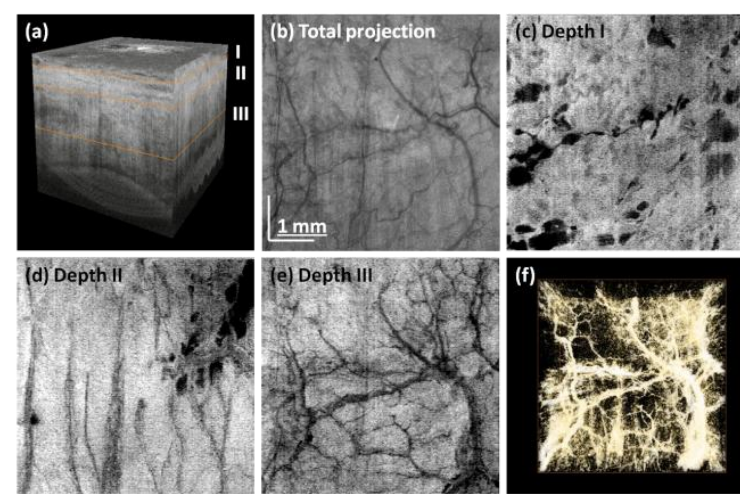

Fig. 4. Three-dimensional reconstruction of human limbus after flattening (a). En face projection of structural images (b). Virtual Cscans (projections) from angiographic data set showing vascular networks in episclera (c) and deeper sclera (d)-(e). Vasculature rendering after thresholding and colorscale inversion (f). Data set was acquired using protocol D.

Finally, we performed large scale imaging of the sclera using a mosaic imaging technique. To do this, 12 separate data sets at different positions were acquired in a serpentine-like sequence. Individual scanning regions were $5.3 \mathrm{~mm} \times 5.3 \mathrm{~mm}$ (protocol D in Table 1), and at least $20 \%$ of overlapping of each edge was assured during the scanning. Individual projections were stitched together to form a final composite projection image.

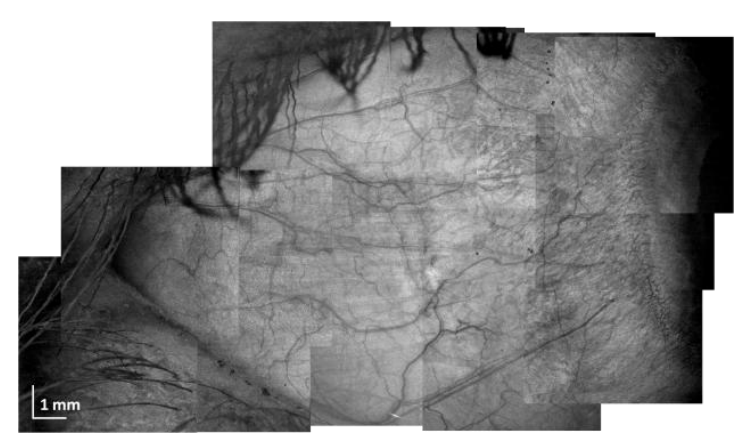

Fig. 5. Large area scleral OCT imaging. Composite projection image from 12 individual data sets acquired using protocol D.

To conclude, we demonstrated in vivo imaging of corneoscleral limbus and sclera using a swept source $1050 \mathrm{~nm}$ system. We have also demonstrated a method for vessel segmentation which is based specifically on structural (intensity) images. This enables qualitative presentation of vascular networks in the anterior segment of the eye. The speed of the system and sensitivity provided by dual-balance detection enabled generation of three-dimensional data sets without significant motion artifacts. Finally, large-scale scleral imaging was performed which allowed for comprehensive visualization of vessels and features in the anterior segment of the eye.

The study has been supported by the National Institutes of Health (R01-EY011289-25, R01-EY013178-11, R01EY01356-06, R01-CA075289-15), Air Force Office for Scientific Research (FA9550-10-1-0551 and FA9550-101-0063). IG acknowledges support from Foundation for Polish Science (KOLUMB Programme; KOL/3/2010-I).

\section{References}

[1] D. Huang et al., Science 254, 1178 (1991).

[2] W. Drexler, J. G. Fujimoto (ed.), Optical Coherence Tomography. Technology and Applications (Springer, Berlin-Heidelberg 2008).

[3] A.M. Zysk, A. L. Oldenburg, D.L. Marks, F.T. Nguyen, S.A. Boppart, J. Biomed. Opt. 12, 051403 (2007).

[4] Y. Wang, A. Fawzi, O. Tan, J. Gil-Flamer, D. Huang, Opt. Exp. 17, 4061 (2009).

[5] M. Pircher, C.K. Hitzenberger, U. Schmidt-Erfurth, Prog. Ret. Eye Res. 30, 431 (2011).

[6] M. Wojtkowski, Appl. Opt. 49, D30 (2010).

[7] J. A. Izatt et al., Arch. Ophthalmol. 112, 1584 (1994).

[8] M. Gora et al., Opt. Exp. 17, 14880 (2009).

[9] B. Potsaid et al., Opt. Exp. 18, 20029 (2010).

[10] Y. Yasuno et al., Opt. Exp. 13, 10652 (2005).

[11] D.R. Anderson, Am. J. Ophthalmol. 108, 485 (1989).

[12] R.K. Wang, L. An, P. Francis, D.J. Wilson, Opt. Exp. 17, 8926 (2009).

[13] B.J. Vakoc et al., Nature Med. 15, 1219 (2009).

[14] K. Bizheva, N. Hutchins, L. Sorbara, A.A. Moayed, T. Simpson, Biomed. Opt. Exp. 2, 1794 (2011).

[15] L. Kagemann et al., Invest. Ophthalmol. Vis. Sci. 51, 4054 (2010).

[16] L. Kagemann et al., Exp. Eye Res., 93, 308 (2011).

[17] P. Li et al., Biomed. Opt. Exp. 2, 3109 (2011).

[18] M.A. Choma, M.V. Sarunic, Ch. Yang, J.A. Izatt, Opt. Exp. 11, 2183 (2003). 